\title{
Research on the impact of block chain technology on China's e-commerce
}

\author{
Tong Zhang, Chunbo Tan
}

Qingdao Huanghai University, Qingdao, China

\begin{abstract}
With the continuous maturity of block chain technology, its application is being carried out in the field of e-commerce. With the properties of disintermediation, strong security, traceability, transparency and non-tampering in the transaction, block chain technology can provide a perfect solution for e-commerce. This paper summarizes the properties and application status of block chain technology, analyzes the problems faced by e-commerce nowadays, introduces the innovative application of block chaining technology, and probes into the influence of block chain technology on e-commerce in China.
\end{abstract}

Keywords: Block chain , E-commerce, Impact

\section{Introduction}

Block chain technology is a new model of disruptive innovation, and it is very likely to set off a new technology and industrial revolution around the world. [1] The United Nations, the International Monetary Fund and the developed countries have paid great attention to the block chain technology, and actively promote the practical application of the block chain technology. The application of block chain technology take the lead in the field of China's financial science and technology, and quickly entered a period of rapid development. [2] With the introduction of "Internet +" concept and the gradual integration of traditional industries and the Internet, the growth of e-commerce market in China tends to be stable, also encountered the bottleneck in progress. The application of block chain technology has extended to the Internet of things, supply chain management, digital asset transaction and other fields related to e-commerce, which will have a profound impact on the development of e-commerce.

\section{The properties of block chain technology and its application in China}

Block chain is the underlying technical architecture of bit-coin, which is a shared, distributed database technology. It makes full use of P2P, shared ledger based on cryptography, intelligent contracts and other mature technology system, and combines many mature technologies together to form a new architecture (or protocol ). [3] It has the properties of decentration, trustless, collective maintenance, reliable database and so on. It can be widely used in various fields.

Currently, there are three development directions for block chain, namely block chain 1.0, block chain 2.0, block chain 3.0. Block chain 1.0 is a virtual currency represented by bit-coin. Block chain 2.0 can be understood as the application of block chain technology in other financial fields. The application of block chain 3.0 covers all aspects of human society. A number of domestic financial science and technology enterprises have begun to explore the financial application scene of block chain technology since 2016. "Big data + block chain" also began to be applied to the warehousing and logistics industry. The application of block chain in notarization, medical service, Internet of things, supply chain, communication and other fields has also been carried out.

\section{The status of the China's electronic commerce and the problems encountered}

The status and trend of the development of e-commerce in China

At present, the e-commerce in China has entered a high-speed growth period, which has caused a great impact on offline entities, and also prompted offline entities to start thinking about changing their business models. The e-commerce develops rapidly , and the market development space will 
be limited.[4] The product of the entity industry is the basis of e-commerce, also the basis of online sales. Under the impetus of sharing economy and shared economy, the traditional industrial chain is constantly integrating with e-commerce. Driven by online traffic, the offline entities and online products are seamlessly integrated to form a win-win ecosphere. As the market is saturated, the online market will not continue to grow.

With the establishment and improvement of mobile e-commerce ecosystem, consumers' online and offline integrated consumption experience needs have been greatly met, and online retail penetration has increased year by year. And the B2B e-commerce market transaction scale growth slowdown, and a steady development trend will form. The rapid development of mobile Internet technology and mobile devices has promoted the mobile shopping market. Users' shopping habits have also been changed. E-commerce has also entered the era of mobile Internet. At the same time, cross-border e-commerce also ushered in a high speed development period.

Problems in the development of e-commerce in China

(1) Regional development is uneven and the gap between enterprises is large

Due to geographical location and economic and social development, the development of China's e-commerce region presents uneven properties. The development of e-commerce in the southeast coastal areas is far superior to the central and western regions. In the same way, the development level of e-commerce is not balanced because of the restriction of management level, capital and talent. The gap between large enterprises and small and medium-sized enterprises is also very large.

(2) The network security is not high, the credit system should be improved

E-commerce is more demanding on the security of the network. In order to improve the competitiveness and improve the user experience, e-commerce companies often collect consumer personal information to analyze and make marketing strategies. The protection of consumer information is not complete, and it often involves information security. The e-commerce develops rapidly, and the hardware infrastructure and the Internet, communication technology update is slow. It also has a certain impact on the network security. The development of e-commerce requires standardized contracts and a perfect regulatory system to reduce or eliminate transaction risks. The current credit system construction still faces a series of problems, such as the isolated island of credit information, the standard of credit evaluation, the credit legislation, the construction of the credit system and so on.

(3) The market supervision is not enough, and the tax collection and management of the ecommerce needs to be strengthened

The infringement of the legitimate rights and interests of consumers often occurs in the ecommerce market because of the mismatch between the regulation and the development of the industry. Under the restriction of technology, the overall supervision of the e-commerce market is backward, which can not meet the demand of market supervision. It is mainly embodied in the information management of online products, the supervision of the transaction service and the protection of the rights and interests of the consumers. With the growing maturity of e-commerce and the increasing proportion in e-commerce, due to its own properties, there is a blind area of tax collection for e-commerce taxation. Therefore, it is necessary and feasible to strengthen the tax collection and management of e-commerce.

(4) The logistics system still needs to be improved, and the talents of e-commerce are lack

At present, the logistics distribution system in China has been preliminarily formed, and the level of logistics management remains to be improved, and the national or terminal logistics and distribution ability is still insufficient. Because China's e-commerce education system is still in the process of exploration, and the e-commerce itself develops rapidly and other factors, the ecommerce talents gap is increasing, and the talent is lack. 


\section{The application prospect and impact of block chain technology in e-commerce}

Application prospect of block chain technology in e-commerce

At present, e-commerce uses centralization services. EBay, Amazon, Alibaba and other trading platforms have restrictive policies and charge platform and transaction costs. This kind of platform can only accept mode of payment that both the seller and the buyer will pay the payment, such as credit cards, PayPal and Alipay. They all need personal information from the customer, which is likely to cause information embezzlement or trafficking, for advertising or other worse uses. Buyers and sellers are not always free to exchange goods and services, as the company and the government review all types of transactions.

A typical representative of the application of block chain in the field of e-commerce is OpenBazaar. OpenBazaar is a new way to buy and sell goods and services online. By running a program on the computer, users can directly connect to other users on the OpenBazaar network and trade with them. The network is not controlled by any company or organization. It is a decentralized peer-to-peer network without the cost of platform use, and the market is anti censorship. Goods and services are traded in OpenBazaar using bitcoins, which are centralized digital encryption currency. The transaction costs on the bitcoin network are very cheap. OpenBazaar is another way of ecommerce. OpenBazaar puts power in the hands of the user, directly linking the buyer to the seller, without centralization of the service. Because there is no middleman in the transaction process, no cost, no one to review the transaction, users can only disclose the selected personal information according to their own wishes. OpenBazaar is an open source project, the code can be open for people to review or audit, and can be open source to improve.

In August this year, the first domestic block chain e-commerce "medium purchase" on-line, using block chain traceability technology to realize the traceability of goods. Ali, Baidu, Tencent, Jingdong and other Internet technology companies are beginning to dabble in the area of block chain. The block chain technology is gradually industrialized and popular, and the block chain technology has already been used in various industries, such as information certification, public welfare assistance, anti-corruption and other fields. The block chain will be a new value transmission network.

The impact of block chain technology on China's e-commerce

Block chain technology will reshape the new model of e-commerce

In the traditional e-commerce mode, the third party payment platform has been introduced to ensure the rights and interests of both parties, which is centralization process, and there are many loopholes. In e-commerce shared trust system based on block chain technology, all participants shared and maintained this trust body. All links were recorded and could be queried. The participation of the participants will be more extensive, and all the buyers will be partly involved in various links from production to procurement. The new model of e-commerce also reduces the cost of building trust. The block chain technology uses a distributed book model, so each transaction can not be tampered with, and it maintains the authenticity of the information on the global network.

Using block chain technology, we can create a high quality, low price e-commerce platform, which can carry out quality evaluation. An intelligent contract for block chains can enable all businesses to achieve seamless cross-border union. Block chain technology can form a decentralized, highly autonomous distributed network platform, enabling transactions in ecommerce market to be completely transparent, authentic and traceable. With the block chain technology, the government's supervision of the e-business market will be more effective.

Block chain technology will rebuild the supply chain ecosystem

Traditional e-commerce supplier supply chain is facing many problems at present, such as supply chain management concept, inventory problem, supply chain integration, supply chain parties' trust, intermediate links and cost problems. And the "block chain+ supply chain" will recreate the supply chain ecosystem to effectively solve the problems above.

Block chain technology can build trust between the parties on the supply chain and make the supply chain more transparent. The transparent supply chain is the ideal state of the purchase link, 
and it can effectively solve the counterfeit and shoddy problem through the product traceability. Trading on the e-commerce network based on block chain technology can effectively reduce transaction costs and track the quality assurance of the entire value chain. With the development of various industries, the block chain technology will be gradually applied to the supply chain system. Customer and government demand for transparency, traceability and speed of transactions will also be higher and higher. Block chain technology is the key to create an e-commerce supply chain ecosystem and eliminate the blind spot of supply chain.

Block chain payment provides a perfect payment solution for cross-border e-commerce

The distributed network built by block chain technology can achieve low cost or even zero cost for cross-border remittances, and can accomplish cross-border transfers at a very fast speed. In addition to the advantages of convenience, fast recording speed and low cost, block chain technology can also be decentralized, with high security and traceablity. Block chain technology can be used to optimize cross-border business and create new cross-border payment methods. The traditional cross-border payment method has a long period of liquidation, higher formalities and frequent cross-border payment fraud, increasing the risk of cross-border capital.

Block chain point-to-point payment removed third party financial institutions, not only can allday payment, instant arrival withdrawals, easy and no invisible cost, it can also help reduce crossborder capital risk and meet the convenience of cross-border payment and settlement services demand.

\section{Conclusion}

E-commerce has changed the traditional "middleman" trade mode, and established a centralized e-commerce platform. The emergence of block chain technology will also change the e-commerce mode and become a new driving force for the development of new e-commerce. The application of block chain technology in the field of e-commerce will effectively solve a series of problems such as regulation, transaction mode, supply chain and so on, and create a new disruptive e-commerce mode.

\section{References}

[1] Tan, Pham Ngoc, Tran Cong Luc, and Nguyen Trung Minh. "Design and Research on HighSpeed Ethernet Information Module." Journal of Applied Science and Engineering Innovation 4.3 (2017): 68-71.

[2] Liu Q. Research and Practice Exploration Into Government's Organization Management in the E-government Environment[J]. Journal of Applied Science and Engineering Innovation, 2016, 3(3): 113-115.

[3] Wang W T, Wang Y S, Liu E R. The stickiness intention of group-buying websites: The integration of the commitment-trust theory and e-commerce success model[J]. Information \& Management, 2016, 53(5): 625-642.

[4] Nie J. Research on Task Scheduling Strategy Based on Cloud Computing Environment[J]. Journal of Applied Science and Engineering Innovation, 2018, 5(1): 9-12. 